\title{
Gl-Blutung am Wochenende wirklich schlechter versorgt?
}

\author{
Ereignet sich eine obere gastrointes- \\ tinale Blutung am Wochenende, so \\ haben diese Patienten keine schlech- \\ tere Prognose als während der \\ Woche, obwohl schwerer kranke Pa- \\ tienten behandelt werden und mehr \\ Zeit bis zur Endoskopie vergeht.
}

- Es ist nicht nur sprichwörtlich, sondern konnte auch für viele Erkrankungen wissenschaftlich erwiesen werden, dass Patienten über das Wochenende im Krankenhaus schlechter versorgt werden als während der Woche. Die Gründe dafür sind vielfältig, doch dürfte der wichtigste in der ausgedünnten Personaldecke und der geringeren Verfügbarkeit von qualifiziertem Personal liegen.

Im Rahmen eines Qualitätssicherungsprojekts wurden die Daten von 6749 Patienten prospektiv gesammelt, die mit einer oberen gastrointestinalen Blutung in 212 englischen Krankenhäusern vorstellig wurden. In einem logistischen Regressionsmodell untersuchte man einen möglichen Zusammenhang zwischen der Einlieferung am Wochenende und der Mortalität. Patienten, die am Wochenende aufgenommen wurden, befanden sich mit größerer Wahrscheinlichkeit im Schockzustand (39\% vs. $36 \%$ ), hatten häufiger eine Hämat-

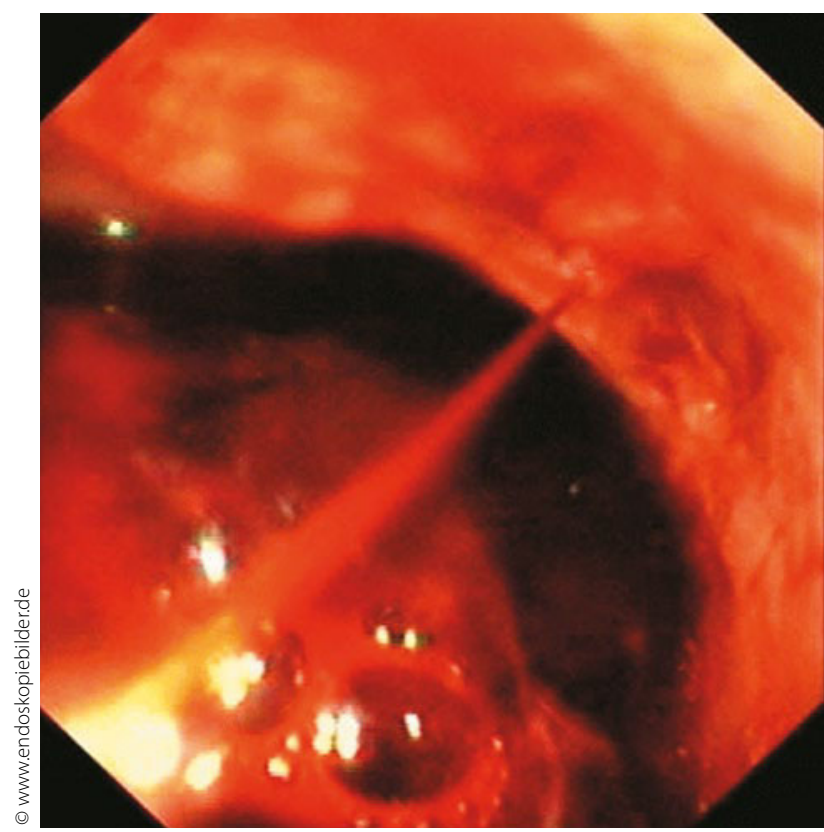

Arterielle Blutung aus einem Magengeschwür. emesis ( $4 \%$ vs. $38 \%$ ) und erhielten häufiger Bluttransfusionen (42 vs. 39\%).

Obwohl der Anteil von Patienten, die eine endoskopische Therapie erhielten, am Wochenende genauso hoch war wie während der Woche (24\%), wurden nur $38 \%$ der Wochenend-Patienten innerhalb von $24 \mathrm{~h}$ nach der Aufnahme endoskopiert. Während der Woche war dies bei $55 \%$ der Fall.

Nach Korrektur für konfundierende Faktoren ergaben sich keine Hinweise auf einen Mortalitätsunterschied zwischen den an Wochentagen und den an Wochenenden behandelten Patienten. Wenn man die Analyse nur auf die Patienten beschränkte, die tatsächlich endoskopiert wurden $(n=5004)$, ergab sich auch hier kein Unterschied in der Prognose. Interessanterweise spielte es für die Mortalität auch keine Rolle, ob das Krankenhaus einen Notfall-Endoskopiedienst vorhielt oder nicht.

Kommentar

Die Untersuchung steht im Widerspruch zu zwei größeren US-amerikanischen Untersuchungen, die bei Patienten mit am Wochenende behandelter oberer gastrointestinaler Blutung eine Mortalitätszunahme von 10$20 \%$ im Vergleich zu den während der Woche aufgenommenen Patienten festgestellt haben. Allerdings verwendeten diese Studien Entlassungsdaten, die nur in geringem Ausmaß die klinische Symptomatik und die Laborbefunde bei Aufnahme berücksichtigen. Die hier vorgestellte, methodisch anspruchs- volle Studie verweist zumindest für die obere gastrointestinale Blutung das Gerücht eines "Wochenend-Effekts" ins Reich des Mythos. Sie bestätigt indirekt auch die Zweifel an der grundsätzlichen Notwendigkeit einer notfallmäßigen Gastroskopie dieser Patienten. Tatsächlich konnte bisher in keiner Studie eindeutig nachgewiesen werden, dass eine frühe Endoskopie die Mortalität beeinflusst. Wichtiger ist wahrscheinlich eine suffiziente intensivmedizinische Behandlung. Für die gastroenterologischen Abteilungen in den
Krankenhäusern wird es aufgrund dieser Daten schwieriger, die Vorhaltung eines endoskopischen Notfalldienstes medizinisch zu rechtfertigen.

H. S. FÜESSL

\section{- V. Jairath et al.}

(NHS Blood and Transplant and Translational Gastroenterology Unit, John Radcliffe Hospital, Oxford OX3 9DU; vipul.jairath@nhsbt.nhs.uk): Mortality from acute upper gastrointestinal bleeding in the United Kingdom: does it display a "weekend effect"? Am. J. Gastroenterol. 106 (2011) 1621-1628 